\title{
Lob der Oberfläche! Der Tod und die Mode in Elfriede Jelineks „Jackie“
}

„Nur die oberflächlichen Eigenschaften dauern“, so Oskar Wilde in seinen Sätzen und Lehren zum Gebrauch der Jugend: „Des Menschen tieferes Wesen

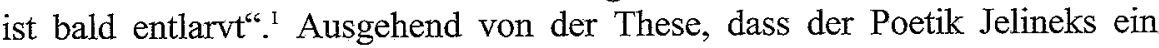
„Lob der Oberfläche“ eingeschrieben ist, möchte ich im folgenden die Rolle der Mode beleuchten, und zwar nicht nur als Oberflächenphänomen, sondern auch als Übergangsphänomen, das an der Schwelle zwischen Oberfläche und Tiefe in Erscheinung tritt.

„Ist die Mode für mich ein Halt, mit dem ich mich auf der Erde fixieren kann, weil ich sonst nichts verstehe?" fragt Jelinek in einem Beitrag über Mode $^{2}$ für die Süddeutsche Zeitung und stellt damit das „Lob der Oberfläche“ in den Dienst einer lebensweltlichen Erdung, bei der Ich-Findung und IchVerlust zusammenfallen:

Von wenig Dingen verstehe ich so viel wie von Kleidern. Ich weiß wenig von mir, interessiere mich auch nicht sehr für mich, aber mir kommt vor, daß meine Leidenschaft für Mode mir mich selbst ersetzen kann" (SZ)

Jelinek argumentiert hier ganz ähnlich wie die Protagonistin ihres Theatermonologs Jackie, ${ }^{3}$ der mittlerweile in einer preisgekrönten Hörspielfassung des Bayerischen Rundfunks vorliegt": „Ich bin meine Kleidung, und meine Kleidung ist ich ${ }^{\text {c5 }}$ stellt Jackie fest - allerdings aus einer keineswegs geerdeten Position: Sie spricht als Tote, die vom Jenseits aus ihr Leben Revue passieren lässt. Indes muss sie sich auch im Jenseits noch modebewusst zeigen: „Jackie sollte in einem Chanel-Kostüm auftreten, denke ich", schreibt die Stückeschreiberin im ersten Satz ihrer Regieanweisung.

Aber warum, so könnte man fragen, warum denkt die Stückeschreiberin Jelinek das?

Doch halt! Darf man dieses „denke ich" tatsächlich der realen Autorin Jelinek zuschreiben, wie ich es gerade getan habe? Muss man hier nicht mit Peter Szondi davon ausgehen, dass der Dramatiker im Drama abwesend ist? ${ }^{6}$ Ande-

1 Wilde, Oskar; Sätze und Lehren zum Gebrauch der Jugend, in: ders. Werke in 2 Bänden, Bd. 1, Berlin o.J., S. 695.

2 Jelinek, Elfriede; „Mode“". Magazin der Süddeutschen Zeitung, März, 2000.

3 Jelinek Elfriede; Jackie", in: dies, Der Tod und das Mädchen I-V. Prinzessinnendramen, Berlin, 2003, S. 63-100.

4 Jackie, BR 2003, Regie: Karl Bruckmaier.

4 Jackie, BR 2003, Regie: Karl Brucko

6 Szondi, Peter, Theorie des modernen Dramas (1880-1950), in: ders., Schriften I. Frankfurt a.M. ${ }^{3} 1989$, S. 17 
rerseits könnte man aber auch argumentieren, dass diese Regieanweisungen und mit ihnen das „denke ich" noch gar nicht „im Drama“" sind, dass Regieanweisungen als Paratexte einen Ort der Rede markieren, der außerhalb oder doch zumindest am Rande des Textes situiert ist. Ich möchte der Frage: „Wer spricht? ${ }^{67}$ nicht weiter nachgehen; und auch die Frage: „Von wo wird gesprochen" will ich nur rasch am Rande streifen.

Paratexte haben, darauf weist Gérard Genette in seinem gleichnamigen Buch hin, eine spezifische Schwellenfunktion. Sie sind, so Genette, eine Art „Vestibül, das jedem die Möglichkeit zum Eintreten oder Umkehren bietet", eine

„unbestimmte Zone' ${ }^{c}$ zwischen innen und außen, die selbst wieder keine feste Grenze nach innen (zum Text) und nach außen (dem Diskurs der Welt über den Text aufweist) ${ }^{\circ .8}$

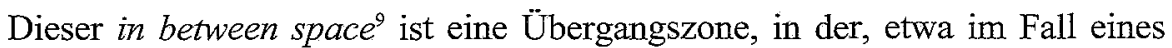
Romanvorworts, der Autor dem Leser durch Leseanweisungen eine Möglichkeit zum Eintreten in den Text eröffnet. ${ }^{10}$

Im Fall von Jackie ist es nun keine Lese-, sondern eine Regieanweisung, mit der die Stückeschreiberin eine modische Direktive zum mise en scéne gibt. Damit adressiert sie jene allmächtige Instanz, die sie in ihrem Theater-Essay „Ich möchte seicht sein“ als „Herrn Regisseur" tituliert:"1 eine Instanz, die dem Dramentext zum performativen Eintreten ,in die Welt" verhelfen wird; eine Instanz aber auch, mit der die Stückeschreiberin am Rahmen ihres Dramentextes und im Rahmen ihrer Regieanweisung ein kursiviertes, Zwiegespräch beginnt: Ein Zwiegespräch, das weniger den Charakter eines direktiven Sprechakts, denn vielmehr den Charakter einer Verhandlung mit einem einflussreichen Gegenüber hat, der am Ende womöglich doch macht, was er will. Ein Zwiegespräch aber auch, das die Stückeschreiberin - und hier zeigt sich eine weitere Parallele zu der Protagonistin ihres Stücks - aus einer quasi jenseitigen Position heraus beginnt: Wissend, dass sie als im Dramentext Abwesende nur eine Randexistenz führen kann und somit im Abseits steht.

An dieser Stelle sei noch auf eine Merkwürdigkeit hingewiesen: Wenn Genette vom Vorwort als einer Art „Vestibül“" spricht, dann vergleicht er es mit einem Vorraum, der als ,unbestimmte Zone' das Eintreten in den Text oder das Umkehren zurück in die Welt ermöglicht. Angesichts der einigermaßen unklaren Etymologie des Wortes „Vestibül“, könnte man freilich spekulieren,

7 Foucault Michel; ${ }^{-}$Was ist ein Autor? (Vortrag) “, in: Dits et Ecrits. Schriften, Erster Band Frankfurt a.M., 2001, S. 1003-1041, hier S. 1003.

8 Genette; Gérard, Paratexte, Frankfurt a.M., 1992, S. 10.

9 Genette; Gérard, Paratexte, Frankfurt a.M., 1992, S. 10.

10 Vgl. Wirth; Uwe, „Das Vorwort als performative, paratextuelle und parergonale Rahmung“, in: Rhetorik. Figuration und Performanz, hg. v. Jürgen Fohrmann, Stuttgart/Weimar, 2004, S. 603-628.

11 Jelinek, Elfriede, "Ich möchte seicht sein", in: Gegen den schönen Schein. Texte zu Elfriede Jelinek, hg. v. Christa Gürtler, Frankfurt a.M., 1990, S. 157-161, hier S. 159. ob hier neben der häuslichen Semantik (vesta) auch eine Kleidersemantik (vestatis) mitschwingt - der Vorraum als Zwischenraum, in dem man seine öffentliche Kleidung ablegt, sich umzieht, bevor man den privaten Raum betritt.

Vor dem Hintergrund dieser (zugegebenermaßen spekulativen) Überlegungen möchte ich zu meiner eingangs gestellten Frage zurückkommen. Warum fordert die Stückeschreiberin im Vestibül des Dramas, dass Jackie in einem Chanel-Kostüm auftreten soll? Warum Chanel? Hinter diesem Satz steht in Klammern:

$$
\begin{aligned}
& \text { "(da müssen Sie aber schon sehr gute Gründe haben, wenn Sie das anders ma- } \\
& \text { chen!) }{ }^{412} \text {. }
\end{aligned}
$$

Bemerkenswerterweise bietet Jelinek im nächsten Satz gleich selbst eine Alternative an: „Man könnte“, so heißt es da „als Vorbild auch dieses letzte Foto im Central Park (mit Maurice Tempelsman), das auf der Bank nehmen. Trenchcoat, Perücke (da Haare durch Chemo ausgegangen), Sonnenbrille und Hermès-Kopftuch ${ }^{\text {“13 }}$. Ja, so geht es auch. Obwohl...

„Sie sollte“, so lesen wir weiter, „sie sollte in jedem Fall schwer arbeiten. Ich stelle mir vor, daß sie ihre ganzen Toten, die Kinder, na, der Embryo und die beiden toten Babies sind nicht so schwer, aber dafür die toten Männer, Jack, Bobby, Telis („Ari“), das ergibt ein ganz hübsches Gewicht, was?!, also, wie soll ich sagen, diese Toten soll sie hinter sich herschleifen wie beim Tauziehen. Oder ein Wolgaschiffer sein Schiff. Das kann ich Ihnen nicht erleichtern. Wenigstens das Blut auf dem rosa Kostüm wiegt nicht allzu schwer, und von Jacks Schädel fehlt sowieso ein ganzes Stück“. ${ }^{14}$

Also doch nicht der Trenchcoat und das Hermès-Kopftuch, sondern das Kostüm. Das rosa Kostüm mit den Blutflecken drauf: Das Kostüm, das Jackie Kennedy am 22. November 1963, dem Tag der Ermordung ihres Mannes in Dallas, getragen hat.

Es ist ein rosa Chanel-Kostüm, das durch ein Ereignis - das KennedyAttentat - Weltruhm erlangt hat und insofern bedeutsam geworden ist. Ein Kostüm von Gewicht, wie man in Anspielung auf Judith Butler sagen könnte. Der Titel von Butlers Buch bodies that matter impliziert, dass Körpern Gewicht zukommt, weil sie Bedeutung erlangen. „,Gewicht verleihen", to matter" - ich zitiere Butler - „,meint [gleichermaßen], ,zu materialisieren“ und ,zu bedeuten “" 15

\footnotetext{
12 Jelinek, „Jackie“, (Anm. 3), S. 65.

13 Ebd.

14 Ebd.

15 Butler, Judith, Körper von Gewicht, Frankfurt a.M., 1997, S. 58.
} 


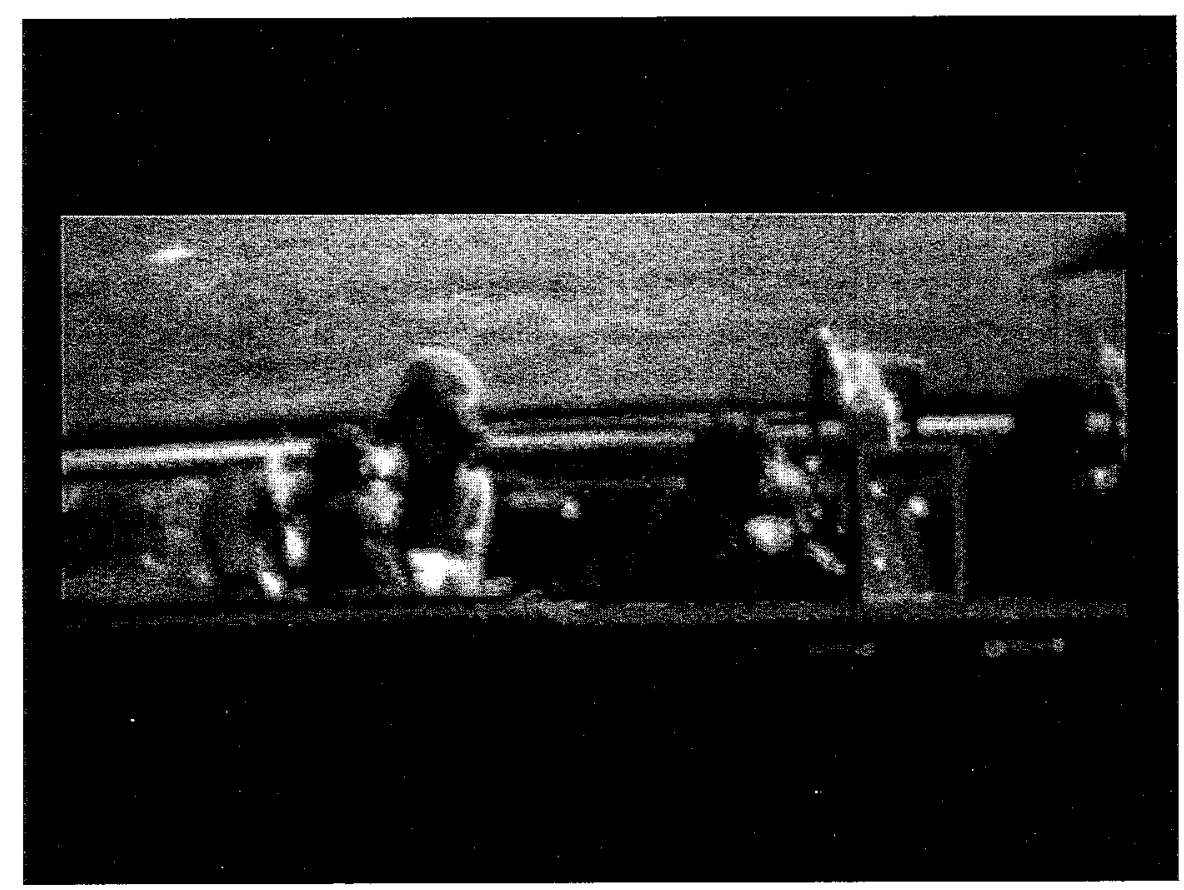

Bild 1: Screen Shot aus dem Film JFK

In Jelineks Jackie erfährt diese Äquivalenz eine Verschiebung: ,zu materialisieren' und ,zu bedeuten ' heißt: sich zu kleiden. Dagegen werden die Körper von Gewicht ausschließlich von den toten Männern Jack, Bobby, Telis bereitgestellt. Natürlich ist auch der Körper der Protagonistin, der Körper von Jackie, ein toter Körper. Aber es ist ein Körper ohne Gewicht: ein Körper, der allein durch die Kleidung bedeutsam, heißt, gewichtig wird.

Wenn ich es recht sehe, dann zeichnet sich Jelineks Theater-Stück Jackie, (das vierte, von insgesamt fünf "Prinzessinnendramen", die unter dem Obertitel Der Tod und das Mädchen stehen) durch eine merkwürdige Interferenz zweier Diskurse aus, denen die Protagonistin ihre Stimme leiht. Ich nenne sie provisorisch den Körperdiskurs und den Kleiderdiskurs. Man könnte aber auch sagen: Der Diskurs des Todes und der Diskurs der Mode. Die Interferenz von Tod und Mode ist, so möchte ich behaupten, das zentrale Motiv des Stücks: ein Stück, das Modenschau und Totentanz zugleich ist. Interferenz bezeichnet dabei sowohl das Moment der Überlagerung und der Störung: Ein Phänomen, dem wir übrigens zu Anfang und Ende der Hörspielfassung von Jelineks Jackie begegnen, wo es zu einer Überblendung von Stimmen kommt: Stimmen, die allesamt von Jackie stammen. Aber auch in der schriftlichen Fassung kommt es zu einer Interferenz zweier Bedeutungsfacetten:
„Also ich markiere mich selbst wie meine Taille, die ich nicht betone. Ich trage unbetonte Kleider. Meine Taille würde durch Betonung erzeugt und gleichzeitig betont, betoniert. Ach so, nein, ich entscheide gerade Wesentliches und entscheide mich anders: meine Taille soll nicht betoniert, sie soll eher angedeutet werden. Sie ist nicht das, was ich an mir besonders hervorheben würde ${ }^{c 16}$.

Betont, betoniert. „Betonen “ heißt hier: Fokussieren der Aufmerksamkeit auf ein Ereignis, aber auch: Ablenken der Aufmerksamkeit. Betonen durch NichtBetonen. „Betonieren“ heißt: Verfestigen eines Zustandes, Herstellen einer Struktur. „Betonen“ und „Betonieren“ werden zum alliterierenden Ausdruck für das Spannungsverhältnis zwischen Ereignis und Struktur: ein Spannungsverhältnis, das, glaubt man Roland Barthes, für die Sprache der Mode von zentraler Bedeutung ist.

Da Roland Barthes in den Regieanweisungen zu Jackie von Jelinek explizit als „Mitarbeiter" genannt wird, ${ }^{17}$ scheint mir ein kurzer Blick in sein berühmtes Buch über die Sprache der Mode gerechtfertigt zu sein: ein Buch, das Barthes 1963 fertig stellte, in dem Jahr also, in dem John F. Kennedy ermordet wurde - und Jackie Kennedys rosa Chanel-Kostüm einen Blutfleck bekam. In dem Kapitel „Varianten der Betonung“ finden wir eine Passage, die als Vorlage für die Anfangssequenz von Jackie gedient haben könnte.

„Insofern sie [die Betonung] das Sein bestimmter Gattungen akzentuiert, einfach indem sie sie nennt" - hier erwähnt Barthes in Klammern den Satz "(Die Taille ist nur angedeutet $)^{\star c}-$ kommt, ich zitiere, ,die Betonung der Existenzbehauptung nahe; man könnte sagen, sie tritt deren Erbe an". ${ }^{18}$

Betonung als Existenzbehauptung - das ist genau die Seinsweise, die Jackie in ihrem Monolog reflektiert; allerdings im inversen Modus: Betonung im Modus der Nichtbetonung: „ich markiere mich selbst wie meine Taille, die ich nicht betone. Ich trage unbetonte Kleider ${ }^{<19}$. Sowohl für Barthes als auch für Jelinek stehen Kleidung und Körper in einem Bedeutungsverhältnis, das durch ein Spiel von Betonung und Nicht-Betonung entsteht. Durch dieses Spiel ermöglicht die Kleidung den Übergang vom Sinnlichen zum Sinn. Der Körper ist, wie Barthes schreibt, „das Signifikat par excellance. Aber welchen Körper soll die modische Kleidung mit Bedeutung versehen?" Beim Versuch, diese Frage zu beantworten, offenbart sich die „strukturale Diskontinuität zwischen Sprache und Sprechen, zwischen Institution und Aktualisierung “. ${ }^{20}$ Und das heißt auch: zwischen Struktur und Ereignis.

Während die Kleidung den Übergang vom Sinnlichen zum Sinn gewährleisten soll, das heißt von konkreten Ereignissen zu einer abstrakten Struktur, steht die Mode vor dem umgekehrten Problem: Sie muss den Übergang vom

16 Jelinek, ,Jackie“, (Anm. 3), S. 66

17 Ebd., S. 65.

18 Barthes, Roland, Die Sprache der Mode, Frankfurt a.M., 1985, S. 123.

19 Jelinek, „Jackie“", (Anm. 3), S. 66

Ebd, S. 264 
abstrakten Körper zum konkreten Körper organisieren. Eine der möglichen Lösungen besteht darin, ich zitiere Barthes, „einen idealen Körper als Inkarnation auftreten zu lassen, nämlich den des Mannequins, des Covergirls“. ${ }^{21}$ Das Covergirl stellt ein seltsames Paradox dar: Einerseits hat sein Körper den Wert einer abstrakten Institution, andererseits ist dieser Körper ein ,individueller“.22

Der Körper des Covergirls muss sich Barthes zufolge ,nach Vorgaben einer gewissen formalen Allgemeinheit, das heißt einer Struktur, richten und ist insofern ,deformiert". Der Körper des Covergirls ist also kein individueller Körper, sondern eine reine Form, die kein Attribut trägt ${ }^{\leftarrow 23}$. Man könnte auch sagen: das Covergirl ist bloße Oberfläche, die medial in Dienst genommen wird. Dabei verweist der Körper des Covergirls durch eine „Art Tautologie“, wie Barthes schreibt, „auf die Kleidung selbst'. ${ }^{64}$ Tautologien sind für Barthes Indizien dafür, dass ein Sinnstiftungsprozess mythologischen Charakter annimmt. Der Mythos Mode zeichnet sich dadurch aus, dass er eine Tautologie zwischen der Kleidung als sinnlichem Signifikant und dem Sinn der Kleidung herstellt.

Während die reale Kleidung praktischen Zwecken dienen muss - Stichwort Schutz, Scham, Schmuck -, entfallen diese Zwecke bei der ,,repräsentierten“ Kleidung ${ }^{625}$ wie man sie beispielsweise in einem Modekatalog sieht. Die Aufgabe einer solchermaßen repräsentierten Kleidung ist es, „Schutz, Scham und Schmuck zu bedeuten ${ }^{\star 26}$. Die Trägerin dieser repräsentierten Kleidung, also das Mannequin oder das Covergirl, wird zu einer Bedeutungsträgerin. Der Körper des Covergirls wird zum Medium der Autopräsentation: Vermittels dieses Körpers bedeutet die Kleidung ,sich selbst. ${ }^{“ 27}$ Dergestalt tritt die Kleidung nicht nur als Signifikant, sondern - wie der Körper - als Signifikat in Erscheinung: Die Kleidung bedeutet etwas im System der Sprache der Mode. Sie ist ein Ereignis, in dem eine Struktur verkörpert wird. Eben dadurch erhält Kleidung ihr semantisches Gewicht.

Zugleich hat Kleidung aber auch ein materiales Gewicht. „Die Techniker der Mode wissen sehr genau“, so Barthes in Die Sprache der Mode, „daß nichts einen Stoff besser definiert als sein Gewicht" ${ }^{28}$ Diese Feststellung mündet kurz darauf in eine Überlegung, die man als Leitmotiv von Jelineks „Philosophie der Mode' deuten könnte. Nach Barthes trägt „die Realität der Kleidung" nämalich, ,poetische Züge":

„Als Substitut des Körpers hat sie durch ihr Gewicht an den grundlegenden Menschheitsträumen teil: am Himmel und an der Hölle, an der Erhebung des

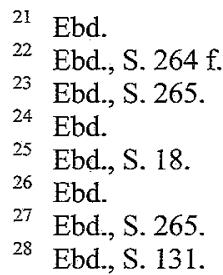

27 Ebd., S. 265.
28 Ebd., S. 131.

Geistes ins Sublime und am Versinken in den Schlaf. Durch ihr Gewicht wird die Kleidung Flügel oder Leichentuch“. ${ }^{29}$

Halten wir vorläufig fest: An die Stelle der Körper von Gewicht treten die Kleider von Gewicht. Das Gewicht der Kleider macht nicht nur die Kleider selbst, sondern auch den Körper, der sie trägt, bedeutsam.

Vielleicht verstehen wir nun etwas besser, was Jackie meint, wenn sie in Jelineks Theaterstück sagt: „Ich bin meine Kleidung, und meine Kleidung ist ich": Das ist ein gewichtiger, ein bedeutsamer Satz, der die Seinsgewissheit des cogito ergo sum und den Absolutismus des l'etat c'est moi in ein tautologisches Lob der Oberfläche verwandelt. Ich kleide mich, also bin ich - meine Kleidung ist ich. Ein Lob der Oberfläche, das aus dém Munde eines CoverGirls kommt, das auf sich selbst verweist, indem es auf seine Kleidung verweist.

Es ist ein rosa Chanel-Kostüm, das durch ein Ereignis - das Kennedy-Attentat - Weltruhm erlangt hat und insofern bedeutsam geworden ist. Ein Kostüm von Gewicht, wie man in Anspielung auf Judith Butler sagen könnte. Der Titel von Butlers Buch bodies that matter impliziert, dass Körpern Gewicht zukommt, weil sie Bedeutung erlangen. "Gewicht verleihen", to matter" - ich zitiere Butler - „meint [gleichermaßen], ,zu materialisieren" und ,zu bedeuten“" ${ }^{\text {" } 30}$ Ein Covergirl, das mit diesem Verweis, das Kleid zum Ersatz seines Körpers macht:

„Mit all meiner kostbaren Kleidung, diesen Haufen und Haufen von Stoff, bestehend aus reinen Grundlinien, von denen aus all die Pässe, die Treibbälle gespielt werden, gut gezielt, aber nur einmal, nein, zweimal wirklich gut getroffen, mit all diesen Fetzen, bald flächig, bald voluminös gebauscht, will ich vortäuschen, ich hätte darunter gar keinen Körper. Obwohl ich den doch, sportlich, straff, muskulös, in dieser neuen Variante, die Sie ab den sechziger Jahren überall kaufen konnten, wenn ihn sich auch nur wenige leisten konnten, präsentierte, sozusagen als gehaltlosen Gehalt präsentierte, in zahllosen Zeitschriften. Im Fernsehn. Im Kino. Ich weiß nicht wo noch überall. Ich wurde betont, das heißt, ich wurde tonangebend. Betonung muß nicht Steigerung sein, wie ich schon sagte, es kann auch Steigerung in der Zurücknahme sein. Immer dezent, das ist wichtig, immer die eigenen Reize spiegeln, sie im Spiegel entstehen lassen, nur ja nicht selber reizvoll sein, dann wäre man ja ein Mensch! Die Erfinderin des schulterfreien Abendkleids und des Minirocks im öffentlichen Dienst. In meinem vorsprachlichen Stadium, das man auch das Kleider-Stadium nennen könnte, also in der Form, in der noch nicht gesprochen wird, die aber schon da ist und auf ibre Aussage vor dem Gericht der Menge wartet, sprachen die Kommentatoren unaufhörlich hinein. Über meine Kleidung hat man geredet, fast noch mehr als über mich, und das heißt was! Die war meine Schrift, meine Kleidung. Meine Kleider waren individueller als meine Sprache, verstehen Sie [... $]^{631}$.

\footnotetext{
29 Ebd.

30 Butler, Judith, Körper von Gewicht, Frankfurt a.M., 1997, S. 58.

31 Jelinek, Jackie", (Anm. 3), S. 77.
} 


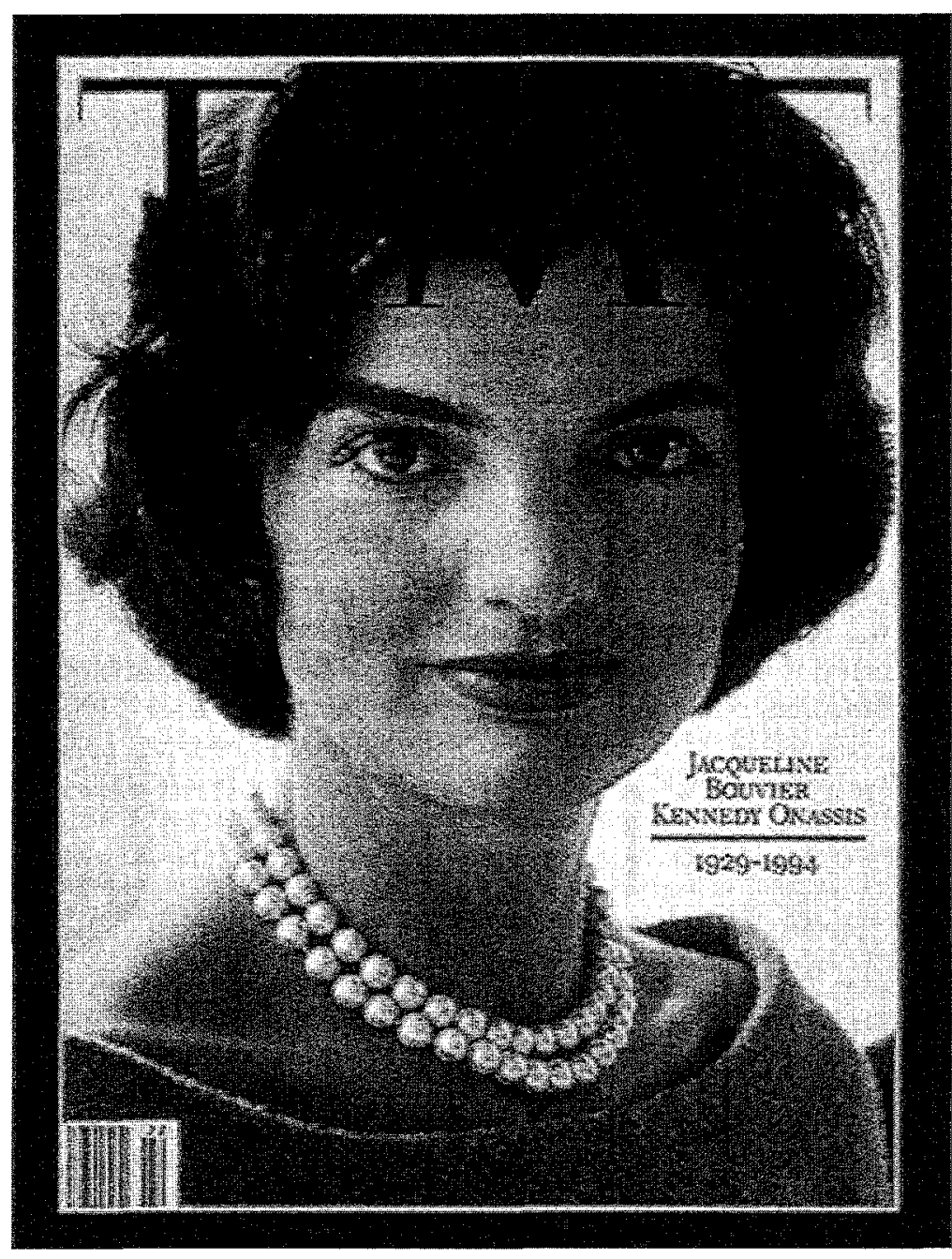

Bild 2: Jackie Kennedy auf dem Cover des Time-Magazine

Hier kommt nun einiges zusammen, und vielleicht kommt auch einiges durcheinander. Hieß es vorhin noch: „Ich bin meine Kleidung, und meine Kleidung ist ich", so scheint es nun mit einem Mal so etwas wie ein Konkurrenzverhältnis zwischen der Kleidung und dem Ich zu geben: „Über meine Kleidung hat man geredet, fast noch mehr als über mich, und das heißt was". Doch nicht nur über die Kleidung wird geredet, sondern die Kleidung redet auch selbst: Jackies Kleidung ist individueller als Jackies Sprache. Das klingt ein wenig nach Wittgenstein: Die Grenzen meiner Kleider sind die Grenzen meiner Welt.
Respektive: Die Grenzen meiner Kleidersprache sind die Grenzen meiner selbst.

Die Frage ist natürlich - und hier setzt Jelinek meines Erachtens eine Interferenz zweier semiologischer Register in Szene - in welcher medialen Form sich die Sprache der Mode äußert: Ist die Sprache der Mode gesprochene Sprache oder ist sie Schriftsprache?

Die Rede vom vor-sprachlichen Stadium scheint auf das Paradigma der Mündlichkeit hinzuweisen. Andererseits lässt ein Satz wie: „Die war meine Schrift, meine Kleidung" keinen Zweifel daran, dass der Stil, sich zu kleiden als Stil des Schreibens vorgestellt wird. Aber warum sollte es überhaupt wichtig sein, sich die Frage zu stellen: gesprochene oder geschriebene Sprache der Mode? Wenn ich es recht sehe, dann sind es zwei Aspekte.

Erstens ist Sprechen immer in irgendeiner Form an die Instanz der Stimme gekoppelt. Und die Stimme ist ihrerseits in irgendeiner Form an einen Körper gekoppelt. Das heißt, die Stimme des Sprechers wirft die Frage nach dem Körper des Sprechers auf: Sie thematisiert das Verhältnis von Körper und Präsenz. Präsenz impliziert Gegenwärtigkeit und Lebendigkeit. Gegenwärtigkeit und Lebendigkeit sind indes flüchtige Phänomene. Die Stimme verhallt. Sie hinterlässt keine Spuren.

Ja, natürlich! Auch die Stimme hinterlässt Spuren. Dann nämlich, wenn sie aufgezeichnet wird: Tonspuren, die mit Hilfe eines Phonographen, eines Tonbands, oder einer CD zu Dauerspuren gemacht werden. Und insofern gibt es, seitdem es audiovisuelle Aufzeichnungsmedien gibt, natürlich auch Stimmen ohne Körper. Stimmen, bei denen die organische Kopplung mit dem Körper durch ein technisches Speichermedium ersetzt worden ist. Allerdings würde ich behaupten, dass aufgezeichnete Stimmen ohne Körper bereits den Regeln des Schriftparadigmas gehorchen.

Und damit bin ich beim zweiten Punkt: Schrift zeichnet sich durch die Möglichkeit der Verdauerung, das heißt, der Speicherung aus. Das bedeutet zugleich: Schrift ist wiederholbar. Sie ist von verschiedenen Menschen, an verschiedenen Orten und zu verschiedenen Zeiten immer wieder aufs Neue lesbar. Nun ist aber Zweifellos auch die gesprochene Sprache wiederholbar. Man kann - etwa als Schauspieler - jeden Abend die gleichen Sätze sagen.

(Auch wenn, wie man hier vielleicht von theaterwissenschaftlicher Seite einwenden könnte, diese Sätze doch nie ganz die gleichen sind. Iterabilité und Alterité - Derrida lässt grüßen ${ }^{32}$ - gehen Hand in Hand)

Worauf es mir in diesem Zusammenhang ankommt, ist jedoch etwas anderes, nämlich dass die Besonderheit der Iterabilität, also der Wiederholbarkeit der Schrift, darin besteht, dass die Schrift die Sprache „vom lebendigen Körper abtrennt" und der Sprache dadurch eine „künstliche Stimme" verleiht, die

32 Derrida, Jacques, „Signatur Ereignis Kontext“, in: ders., Limited Inc, hg. v. Peter Engelmann, Wien, 2001, S. 15-45, hier S. 24 
den Körper zeitlich „überdauern kann“. ${ }^{\text {3s }}$ Schrift muss, „um zu sein, was sie ist" auch in der Abwesenheit des Senders funktionieren können. Heißt: Schrift funktioniert auch dann noch als Kommunikationsmedium, wenn der Sender bereits gestorben ist. Ja, sie funktioniert sogar, wenn der Empfänger tot ist. ${ }^{34}$

Aber was hat das alles mit der Sprache der Mode respektive mit Jelineks Theaterstück Jackie zu tun? Ich denke, es sind im Wesentlichen zwei Punkte.

Erstens scheint es mir so zu sein, dass die Sprache der Mode, wie ja auch jede natürliche Sprache, wahlweise als mündliche und als schriftliche Sprache verkörpert werden kann. In Jelineks Theater-Stück scheint es nun zu einer merkwürdigen Interferenz zwischen Schrift und Stimme zu kommen: Hier wird der Schrift der Mode eine Stimme verliehen.

Wenn es nun stimmt, dass Sprechen an die Präsenz von Stimme gekoppelt ist und wenn es weiter stimmt, dass Jelineks Jackie als Tote zum Publikum spricht, dann muss man sich fragen, ob diese Stimme aus dem Jenseits nicht als eine besondere Art von Schrift - als écriture performatif - aufzufassen ist:

Als theatrale Telegraphie aus dem Totenreich. In dieser Hinsicht - und damit bin ich beim zweiten Punkt - ist das Verhältnis von Schrift und Stimme auch für das Theater von entscheidender Bedeutung. In Jelineks Jackie wird so möchte ich behaupten - die Sprache der Mode als écriture performatif auf die Bühne gebracht, wobei sie freilich einen Medienwechsel erfährt: Sie wird als Sprechakt in Szene gesetzt: rezitiert von einer Stimme ohne Körper - bzw. von einer Stimme mit Körper ohne Gewicht. Wie beim modegerechten Körper des Covergirls fallen in dieser Stimme Struktur und Ereignis zusammen. ${ }^{35} \mathrm{Um}$ was für eine Struktur handelt es sich dabei? Wenn ich es recht sehe, dann handelt es sich um eine Äquivalenzstruktur. In Jelineks Jackie wird eine Äquivalenz zwischen Schrift und Mode einerseits sowie Tod und Mode andererseits in Szene gesetzt. Die Mode ist, wie Walter Benjamin im Passagenwerk feststellt, der "dialektische Umschlagplatz [...] zwischen Lust und Leiche“. Dabei tritt der Tod als - ich zitiere - "flegelhafter Kommis" der Mode in Erscheinung, der - Zitat - „wegen der Ersparnis selbst den Mannequin $[\text { macht }]^{4: 36}$

Indem der Tod dergestalt zum Handlungsgehilfen der Mode wird, erhält die Mode eine Struktur bestimmende Funktion in Benjamins dialektischem Bild. Damit nimmt Benjamin zugleich eine bemerkenswerte Akzentverschiebung vor, denn in Giacomo Leopardis allegorischem Dialogo della Moda e della Morte, den Benjamin seinen Überlegungen über die Mode voranstellt, ist es die Mode, die dem Tod zuarbeitet.

33 Assmann, Jan und Aleida, Lemma „Schrift", in: Reallexikon der deutschen Literatur. Neubearbeitung des Reallexikons der deutschen Literaturgeschichte. Bd. III., hg. v. Jan-Dirk Müller u.a., Berlin/New York, 2003, S. 393-399.

${ }^{34} \mathrm{Vgl}$. Derrida, Signatur, Ereignis, Kontext", (Anm. 31), S. 24.

35 Barthes, Die Sprache der Mode, (Anm. 18), S. 266.

36 Benjamin, Walter, Das Passagen-Werk", in: Gesammelte Schriften, Bd. V.1, Frankfurt a.M. 1991, V.1, S. 111.
Ich sage ${ }^{\text {" }}$, so spricht die Mode bei Leopardi, „daß es unser beider Natur und Gewohnheit ist die Welt fortwährend zu erneuern; du hast dich aber von Anfang an auf die Menschen und das Blut gestürzt; ich dagegen begnüge mich meist mit den Bärten, den Haaren, dem Hausrat, den Palästen und dergleichen Dingen $[\ldots]^{6.37}$

Im weiteren Verlauf des Dialogs wird dann deutlich, inwiefern die Mode die Handlungsgehilfin des Todes ist.

Zu deinen Gunsten habe ich nach und nach vor allem aber in letzter Zeit, die Anstrengungen und Übungen, die dem körperlichen Wohlbefinden nützlich sind, veralten und vergessen lassen [...]. Zudem habe ich in der Welt Sitten und Gebräuche verbreitet, durch die das Leben selbst, in bezug auf Körper und Geist, mehr tot als lebendig wird“.38

Bei Leopardi schwächt die Mode dèn Körper: Das Leben nach der Mode ist ein Leben zum Tode. Es ist ein Leben, das sich - vermittelt durch die Mode permanent im Übergang zum Tod befindet. Bei Benjamin ist es genau umgekehrt: Bei ihm übernimmt der Tod eine vermittelnde, eine mediale Funktion im System der Mode: Er wird zum Mannequin. In beiden Fällen wird die Mode zum dialektischen Umschlagplatz zwischen Körper und Kleidung: „Denn nie war Mode“, heißt es bei Benjamin, „anders als die Parodie der bunten Leiche, Provokation des Todes durch das Weib und zwischen geller, memorierter Lache bitter geflüsterte Zwiesprach mit der Verwesung ${ }^{639}$. Das dialektische Moment dieser Allegorie besteht darin, dass der Tod auf die Provokation des Weibes mit einem performative joke antwortet: er tritt als Mannequin auf. Vielleicht sollte man vor dem Hintergrund des bisher Gesagten, Barbara Vinkens These, Mode sei immer schon eine Form des Crossdressing, ${ }^{40}$ eine existential-ontologische Wendung geben: Mit Blick auf Benjamin muss man nämlich feststellen, dass auch der Tod ein Cross-Dresser ist: Er tritt im Kostüm der Mode auf und verwandelt so den Totentanz in eine Modenschau. Umgekehrt, verkuppelt' die Mode den lebendigen Leib mit der anorganischen, der toten Welt. Das heißt, die Mode operiert an der Schwelle zwischen Leben und Tod: Sie gefällt sich in einem liminalen Cross-Over, das, um noch einmal mit Benjamin zu sprechen, den „sex-appeal des Anorganischen $^{641}$ in Szene setzt.

Meine These wäre nun, dass in Jelineks Theaterstück Jackie die Bühne zum "dialektischen Umschlagplatz" zwischen Tod und Mode gemacht wird - zu einem space inbetween, in dem - verkörpert durch eine dramatis persona,

37 Leopardi, Giacomo, „Dialog zwischen der Mode und dem Tod“ („Dialogo della Moda e della Morte“, (1824)), in: Operette Morali, zit. nach id. Gesänge, Dialoge und andere Lehrstücke, München, 1978, S. 288-292

$38 \mathrm{Ebc}$

39 Benjamin, Das Passagen-Werk, (Anm. 35), S. 111

Vinken, Barbara, „Transvestie - Travestie. Mode und Geschlecht", in: Ästhetik Erfakrung hg. v. Wlad Godzich/Jörg Huber, Heidelberg, 2004, S. 57-76, hier S. 67.

${ }^{41}$ Benjamin, Das Passagen-Werk, (Anm. 35), S. 130 
nämlich Jackie Kennedy - zwei Stimmen zu Wort kommen: Jackie verleiht nicht nur der Sprache der Mode eine Stimme, sondern sie tritt auch als Stimme des Todes auf: In Gestalt eines toten Covergirls, das ein rosa ChanelKostüm trägt. Aus dem männlichen Mannequin bei Benjamin ist bei Jelinek eine Frau geworden, die die Provokation des Todes durch das Weib als Interferenz von Kleiderdiskurs und Körperdiskurs zur Schau stellt.

Indem Jackie die Mode und den Tod verkörpert, wird sie zu einer Allegorie im Doppelpack, zu einer dialektischen Prosopopöie $\mathrm{i}^{42}$. Mehr noch: Sie wird zu einer Verkörperung der strukturellen Äquivalenz von Mode und Tod. Aus dieser Position hält Jackie als "Schwellenfigur zwischen ,Leben“ und ,Tod"cc43 Zwiesprach mit der Verwesung. Die Position im ,Dazwischen' ist deshalb eine Verkörperung der strukturellen Äquivalenz von Mode und Tod, weil die Mode, wie Silvia Bovenschen in ihrem Essay über die „Listen der Mode“ feststellt, die „Permanenz des Wechsels" bezeichnet. $^{44}$

In diesem Zusammenhang begegnen wir interessanterweise noch einmal der Dialektik von „Betonung“ und „Betonierung“. Das modische Prinzip des permanenten Wechsels bricht die Tendenz zur Verfestigung - zur Betonierung immer wieder auf. Der „Bruch mit der Vergangenheit“ den die Mode mit jeder neuen Saison vollzieht, impliziert, wie Simmel in seiner Philosophie der Mode schreibt, eine Betonung der Gegenwart: „Diese Betonung der Gegenwart ist ersichtlich zugleich Betonung des Wechsels ${ }^{6 .}{ }^{45}$ Allerdings ist der Bruch mit der Vergangenheit auch ein Hinweis auf die Vergänglichkeit, die mit dem Wechsel einhergeht. Und eben darin sind sich Mode und Tod gleich. Beide folgen dem Prinzip des ewig erneuten Vergehens. Das memento mori findet seinen Ausdruck in der Erkenntnis: Mensch, gedenke, dass Du Deine Kleider wechseln musst! und wird demgemäß zu einem Memento Mode.

Dergestalt verwandelt sich die vermeintliche Tiefe des Todes in ein Oberflächenphänomen. Zugleich werden die Kleider zu einem Schwellenphänomen zwischen Leben und Tod: Sie markieren einen space in between zwischen dem Reich der Lebenden und dem Reich der Toten. Diesem Dazwischen eignet indes nichts Tiefes oder tiefsinniges - es handelt sich um etwas, das offen zu Tage liegt:

„Mehr als gezeigt wird, kann man nicht wissen. Die Kleider sind absolut tot, obwohl sie an mir zu leben scheinen. Oder lebe ich nur durch meine Kleider? Egal. Es ist jedenfalls eine ganz besondre Eigenschaft. Ich weiß nur nicht ob von mir oder von den Kleidern. Auf den Fotos dann wieder: tot. Man kann es ahnen,

${ }_{42}$ Menke, Bettine, „Die Ver-Stellung und die schöne Stimme: Zum Konzept eines dekonstruktiven Feminismus", in: Raum und Verfahren. Interventionen 2: Museum für Gestaltung Zürich, hg. v. Jörg Huber/Alois Martin Müller, Frankfurt a.M., 1993, S. 65-88, hier S. 67 f.

43 Annuß, Evelyn, Elfriede Jelinek. Theater des Nachlebens, München, 2005, S. 23.

44 Bovenschen, Silvia, „Über die Listen der Mode“", in: Die Listen der Mode, hg. v. Silvia Bovenschen, Frankfurt a.M., 1986, S. 10-30, hier S. 13.

45 Simmel, Georg, „Philosophie der Mode“, in: ders., Gesamtausgabe Bd.10. Frankfurt a.M., 1997, S. 9-37, hier S. 17. was sie bewegt. Daß es eine Frau ist, die sie bewegt. Deshalb habe ich mich so für Mode interessiert. Sie ist was sie ist. Und darin verschwindet der Mensch. Auf meinem Kostüm sind Blut und Gehirnfetzen, aber an das rosa_Kostüm wird man sich erinnern, weil es ein Kostüm ist, in dem mein Geist steckt, dieser unaufhörlich Lernende, bis er selbst Lektor werden darf. In einem Buchverlag. Aus einem Bücherdepot wurde damals ja auch geschossen ${ }^{<46}$

Hier wird die Kleidung in einem zweifachen Sinn als Medium ins Spiel gebracht. Erstens als mediale Oberfläche, auf der alles gezeigt wird, was man wissen kann. Damit wird auf ein strukturelles Wissen um die Permanenz des Wechsels zwischen belebt und unbelebt verwiesen. Zweitens kommt die Kleidung aber auch noch in anderer Hinsicht als mediale Oberfläche ins Spiel, nämlich als Medium der Erinnerung, das die Spuren bedeutsamer Ereignisse Blut und Hirn - als Einschriften speichert.

So besehen tritt die Sprache der Mode als doppelte Schrift in Erscheinung: Zum einen als Prozess des Schreibens (écriture), als écriture performative, die die Permanenz des Wechsels als Wiederholung vollzieht, und zwar so, dass sich die Sprache „vom lebendigen Körper abtrennt" und ihr dadurch eine „künstliche Stimme“ verliehen wird. ${ }^{47}$ Zum anderen als Geschriebenes (écrit), als Monument, an dem sich Spuren von im wahrsten Sinne des Wortes ,merkwürdigen' Ereignissen zeigen.

Indem die Mode ist, was sie ist, tritt sie nicht nur als Oberfläche, sondern auch als Spielfläche in Erscheinung, auf der Zeichen arrangiert und inszeniert werden. Dies ist nun keine besonders originelle Einsicht, denn jede Modenschau, und zwar gleichgültig, ob sie als eine Verkaufsveranstaltung oder als gesellschaftiches Ereignis betrachtet wird, ist eine Perfomance und hat insofern theatralen Charakter. Das Interessante mit Blick auf Elfriede Jelineks Theater-Stück Jackie, ja, mit Blick auf ihre Theater-Poetik überhaupt, ist nun aber, dass die Modenschau zum Modell für das Theater wird. Die Engführung von Mode und Tod einerseits, sowie von Mode und Schrift andererseits, mündet in eine Theater-Poetik, die den Schauspielerkörper in den abstrakten Körper eines Mannequins verwandeln will. So fordert Jelinek in ihrem Essay „Ich möchte seicht sein", dass der Körper des Schauspielers nicht mehr als Ausdrucksmedium ins Spiel kommen soll, dass man von dem Gesicht und dem Körper des Schauspielers nichts mehr „ablesen“ kann, sondern dass die Schauspieler - (ähnlich wie im epischen Theater) - ohne Körper „Sätze sprechen":

"Vielleicht eine Modenschau, bei der die Frauen in ihren Kleidern Sätze sprechen. Ich möchte seicht sein! Modeschau deswegen, weil man die Kleider auch allein vorschicken könnte". 48

46 Jelinek, „Jackie“, (Anm. 3), S. 79 f.

47 Assmann, Lemma "Schrift", (Anm. 32).

48 Jelinek, „Ich möchte seicht sein", (Anm. 11), S. 157 f. 
Mit diesem Postulat organisiert Jelinek das Verhältnis zwischen dem körperlichen Auftritt des Schauspielers im Theater und der Prosopopöia in einer Weise, die „der Belebung der Figurenrede durch den Schauspielerkörper eine Absage erteilt" ${ }^{49}$ In eben dieser Hinsicht scheint mir Jackie eine ideale Verkörperung der Jelinekschen Theaterpoetik zu sein: Eine Theaterpoetik, die am liebsten ohne den Körper auskäme, den Körper durch die Kleider ersetzen würde. Jackie ist der Versuch, den dramatischen Körperdiskurs in einen postdramatischen Kleiderdiskurs zu überführen, und so das Paradox des Schauspielers in ein Paradox des Covergirls zu verwandeln. Diderot behauptete, der Schauspieler dürfe nicht selbst empfindsam sein, wenn er Empfindsamkeit darstellt. Vielmehr diene dem Schauspieler der (ich zitiere) „empfindsame Menschen [...] als Modell ${ }^{\star 50}$. Bei Jelinek wird der Schauspieler zum Model, er - oder sie - soll nicht mehr mit Hilfe seines Körpers ein abstraktes Modell verkörpern, sondern dem Text als - und hier wird es paradox - als quasi körperlose Instanz eine Stimme verleihen. Dergestalt wird aus dem Sprechtheater ein Schrift-Theater, in dessen Rahmen - quasi als „Theatrographie ${ }^{\star 651}$ - eine écriture performatif zur Erscheinung kommt: Eine Art post-mortales Theater, das die Sprache, vom lebendigen Körper abtrennt ${ }^{c}$ und ihr dadurch eine ,künstliche Stimme" verleiht, die den Körper zeitlich überdauern kann.

In eben diesem Sinne erweist sich Jelineks Theater-Monolog Jackie als ein Theater des Nachlebens: ein Theater, in dem nicht nur die Dramatiker abwesend sind, sondern in dem sogar die dramatis personae abwesend werden sollen; ein Theater aber auch, das als Theater des Nachlebens mit dem so genannten wirklichen Leben interferiert.

Von vielen Beobachtern wurde die Tatsache als skandalös empfunden, dass Elfriede Jelinek nicht in persona zur Verleihung ihres Nobelpreises nach Stockholm fuhr, sondern ihre Dankesrede stattdessen als Videoaufzeichnung nach Schweden schickte.

Diese signifikante Abwesenheit der Preisträgerin Jelinek bei der Preisverleihung ist im Lichte ihrer eigenen Theater-Poetik nur konsequent. "Seicht sein" im Sinne Jelineks bedeutet nämlich nicht nur, dass Schauspieler in ihren Kleidern Sätze sprechen, sondern auch, dass sie als Inventar aus „unserem Leben" ausgeschlossen werden, dass sie nur noch als Oberflächen anwesend sind, dass sie als Abwesende sprechen: „Klopfen wir sie platt zu Zelluloid!“ fordert Jelinek, „Wir machen vielleicht einen Film aus ihnen", aber, wie sie hinzufügt, „ein Film als Theater nicht ein Film als Film! $!^{\star 52}$

Annuß, Elfriede Jelinek. Theater des Nachlebens, (Anm. 42), S. 24

50 Diderot, Denis, „Das Paradox über den Schauspieler" (1769), in: ders.: Ästhetische Schriften, Bd. 2, hg. v. Friedrich Bassenge, Frankfurt a.M., 1968, S. 481-538, hier S. 485.

Vgl. hierzu: Theatrographie. Heiner Müllers Theater der Schrift, hg. v. Günther Heeg/Theo Girshausen, 2008

52 Jelinek, Ich möchte seicht sein“, (Anm. 11), S. 160.

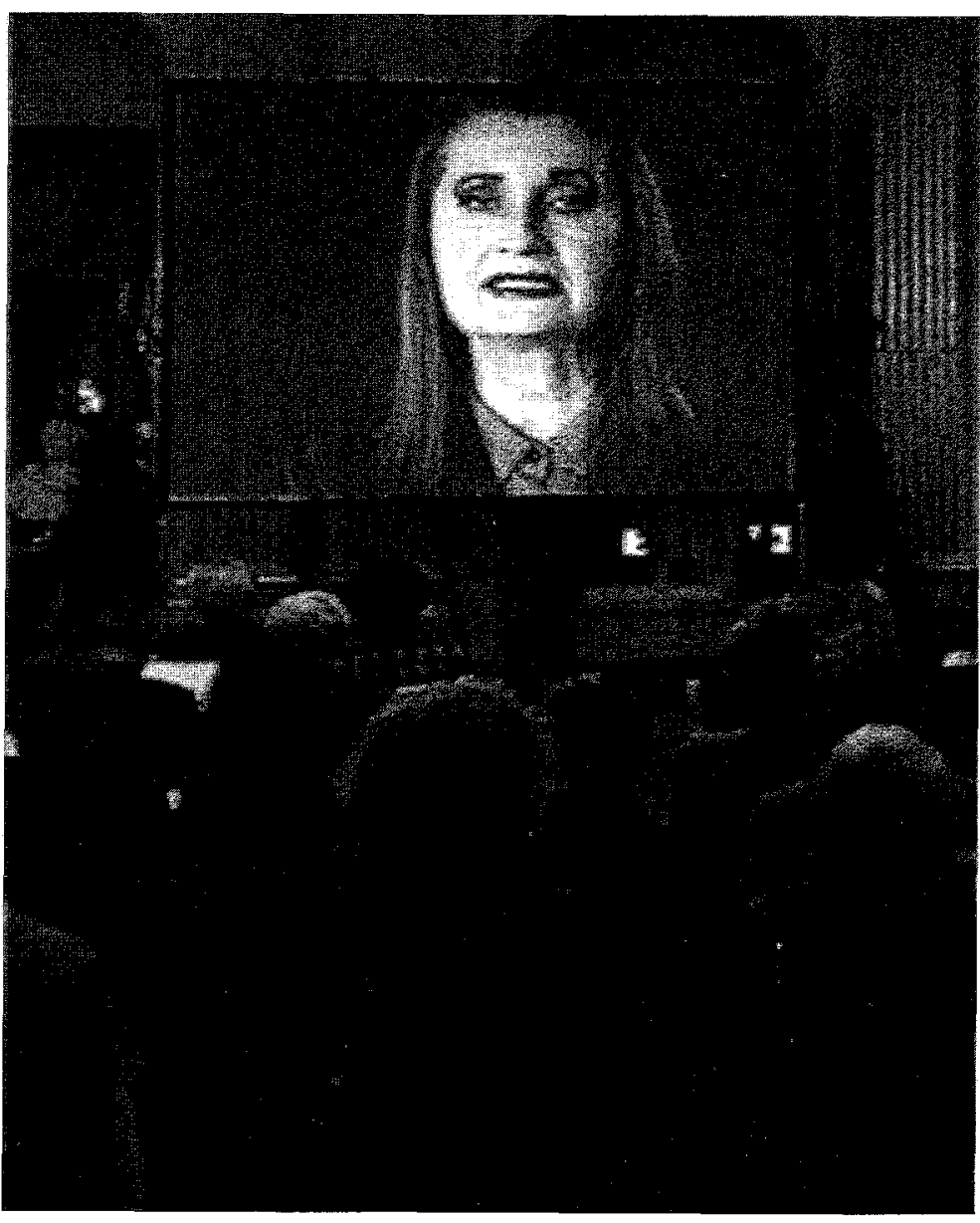

Bild 3: Jelinek bei der Verleihung des Nobelpreises

So besehen lässt sich Jelineks Video-Perfomance bei der Nobelpreisverleihung als Selbstversuch im Rahmen ihrer eigenen Theater-Poetik deuten: als Inszenierung einer Poetik der performativen Nicht-Präsenz, mit der sich die dramatische Schriftstellerin in eine ,unbestimmte Zone' manövriert hat, die zwischen Jenseits und Abseits changiert: eine Zone, aus der heraus sie als Abwesende - plattgeklopft zu Zelluloid, und das heißt: als Körper ohne Gewicht - mit den Anwesenden Zwiesprache hält 